\title{
Near-IR transparent conductive amorphous tungsten oxide thin layers by non-reactive radio-frequency magnetron sputtering
}

\author{
Hao Chen $^{1,2^{*}}$, Alessandro Chiasera ${ }^{3}$, Cristina Armellini ${ }^{3}$, Giorgio Speranza ${ }^{3,4}$, Stefano Varas $^{3}$, Osman Sayginer $^{3}$, Antonio \\ Alfano $^{2}$, Marco Cassinelli ${ }^{2}$, Mario Caironi ${ }^{2}$, Raffaella Suriano ${ }^{5}$, Mohamed Zaghloul ${ }^{1,2}$, Alberto Tagliaferri ${ }^{1,2}$, Maurizio \\ Ferrari $^{3}$ and Silvia M. Pietralunga ${ }^{2,6}$ \\ ${ }^{1}$ Politecnico di Milano, Department of Physics, Piazza Leonardo da Vinci, 32, 20133 Milano, Italy \\ 2CNST@PoliMi, Istituto Italiano di Tecnologia (IIT), Via Giovanni Pascoli 70/3, Milano, Italy \\ ${ }^{3}$ IFN-CNR CSMFO Laboratory and FBK Photonics Unit, Via alla Cascata 56/C Povo 38100 Trento, Italy \\ ${ }^{4}$ Fondazione Bruno Kessler (FBK) - Centro Materiali e Microsistemi (CMM), Via alla Cascata 56/C, 38123 Trento, Italy \\ ${ }_{5}^{5}$ Dipartimento di Chimica, Materiali e Ingegneria Chimica "Giulio Natta", Politecnico di Milano, Piazza L. da Vinci, 32, 20133 Milano, \\ Italy \\ ${ }^{6}$ Institute for Photonics and Nanotechnologies (IFN)—National Research Council (CNR), Piazza L. da Vinci, 32, 20133 Milano, Italy
}

\begin{abstract}
Key assets for transparent electric contacts in optoelectronic applications are high conductivity and large transparency over extended spectral range. Indium-Tin-Oxide and Aluminium-doped-Zinc-oxide are commercial examples, with their electrical conductivity resembling those of metals, despite, their transparency being limited up to $1.5 \mu \mathrm{m}$. This work introduces smooth and compact amorphous thin films of $n$-type semiconducting $\mathrm{WO}_{3-x}$ prepared by RF-sputtering followed by annealing in dry air, as optical layers of tailorable dielectric properties. We evaluate Figure of Merit, combining electrical conductivity and optical transparency, and rate the performances as a transparent conductive layer.
\end{abstract}

\section{Introduction}

The demand for simultaneous light transmission and electrical conduction in thin films, namely transparent conductive films (TCFs), is dramatically increasing among advanced applications such as information displays (e.g. liquid crystal displays [1] and organic lightemitting diodes [2]), and energy technologies (e.g. photovoltaics [3] and architectural/window glass [4]). Transparent and conductive metal oxides (TCOs) are the representative category that is well-studied and widespread in industry [5]. Indium tin oxide (ITO) [6], an alloy of indium oxide $\left(\mathrm{In}_{2} \mathrm{O}_{3}\right)$ and tin oxide $\left(\mathrm{SnO}_{2}\right)$, is probably the most outstanding and well-known TCO, due to the excellent performance at high optical transmittance ( $>85 \%$ ) combined with low electrical resistance (sheet resistance $R_{s}$ ranging from 5 to $100 \Omega \cdot \mathrm{sq}^{-1}$ ). However, some inherent shortcomings, such as high cost of raw material and production, fragile nature in lifetime caused either by mechanical cracking at low strain, or by degradation upon cyclic operation, somehow hinder the sustained use of ITO [7]. Besides, optical transparency of ITO and of other popular TCOs, such as aluminiumdoped and gallium-doped zinc-oxide (AZO and GZO) [8] and fluorine-doped tin oxide (FTO) [9] is frustrated in the Near-InfraRed (NIR) range, where free-carrier absorption arises.
Research into alternative TCOs is therefore becoming necessary. Studies have been dived into organic-based materials and metallic nanostructures, etc. [10], but they seldom reach the ideality for low-cost commercial fabrication and long-lifetime duration. In this study, we analyse amorphous tungsten oxide $\left(\mathrm{WO}_{3-x}\right)$ thin films, prepared by Radio-Frequency (RF) magnetron sputtering followed by thermal annealing in dry air, as an additional choice to the existing TCFs, especially towards the NIR spectral range. Among the various thin-film deposition methods, RF-sputtering has the advantage of cost-effectiveness and upgradability at industrial production scale, while being a flexible, controllable and stable process [11].

Tungsten oxide is a versatile wide bandgap semiconductor, featuring wide polymorphism and compositional ratios. Different stoichiometries and stable crystalline phases have been found, depending on growth conditions [12-15]. In particular, oxygen deficient $\mathrm{WO}_{3-x}$ phases can show remarkable electrical conductance in the presence of considerable optical transparency $[16,17]$. In this experiment, we introduce sub-stoichiometric amorphous $\mathrm{WO}_{3-x}$ thin films deposited by RF-sputtering, that show good optical transparency in the NIR range. We rate their electrical conductivity and discuss their performances as transparent and conductive layers in the NIR.

\footnotetext{
* Corresponding author: hao.chen@polimi.it
} 


\section{Film fabrication and testing}

Amorphous tungsten oxide $\mathrm{WO}_{3-x}$ thin films were deposited by non-reactive RF magnetron sputtering in $\mathrm{Ar}$ atmosphere $[18,19]$, onto different substrates, namely $\mathrm{p}$ doped $\mathrm{Si}$ (100), fused silica $\left(\mathrm{SiO}_{2}\right)$ and ITO-coated borosilicate glass $\left(R_{S}=8 \sim 12 \Omega \cdot \mathrm{sq}^{-1}\right)$. Before deposition, substrates were thoroughly cleaned, by ethanol rinsing followed by heating at $120{ }^{\circ} \mathrm{C}$ for 30 minutes, while the target of stoichiometric $\mathrm{WO}_{3}$ was pre-sputtered for 10 minutes. During deposition, substrates and target were constantly kept at room temperature (RT), and a working pressure at $5.4 \times 10^{-3}$ mbar was stabilized in the deposition chamber.

By changing the deposition time, two sets of film were obtained, respectively about $160 \mathrm{~nm}$ and $50 \mathrm{~nm}$ thick. Post-growth annealing was carried out in a conventional tubular oven [19] for 8 hours in dry air at $300^{\circ} \mathrm{C}$, below the threshold for crystallization [20]. The process provides four film systems to be tested, each one deposited on all the three substrates. Thicker and thinner sets are respectively $\{\mathrm{S} 1, \mathrm{~S} 2\}$ and $\{\mathrm{S} 3 \mathrm{~S} 4\}$, being $\mathrm{S} 1$ and $\mathrm{S} 3$ as-deposited, and $\mathrm{S} 2$ and $\mathrm{S} 4$ annealed.

Thickness $d(\mathrm{~nm})$ and morphology of the films were characterized by High-Resolution Scanning Electron Microscopy (HR-SEM) and profilometry. Surface topology and roughness $R_{a}(\mathrm{~nm})$ were analysed by Atomic Force Microscopy (AFM). The amorphous structure of the films deposited on $\mathrm{Si}$ substrate was assessed by grazing-index X-Ray Diffractometer (XRD) $\theta-2 \theta$ measurements in Bragg-Brentano geometry. The stoichiometry at surface was analysed by X-ray Photoelectron Spectroscopy (XPS). The semiconducting self-doping $n$-type character of the films was revealed by the hot probe method [21]. DC sheet resistance $R_{s}\left(\Omega \cdot \mathrm{sq}^{-}\right.$ 1) was tested at RT by 4-probe method in Van der Pauw configuration [22] on samples deposited on $\mathrm{SiO}_{2}$ substrate. Electrical conductivity was correlated to the position of the Fermi level $E_{F}$ and to the value of work function $(\Phi)$ by Kelvin Probe (KP) measurements (KPSP020, KP Technologies Inc).

Optical absorbance spectrum in the range $300 \mathrm{~nm}$ $2500 \mathrm{~nm}$ was measured on samples deposited onto $\mathrm{SiO}_{2}$ substrate and the amplitude of the optical bandgap $E_{g}(\mathrm{eV})$ was estimated by detailed Tauc plot analysis [23] to an accuracy of $10 \mathrm{meV}$. The complex dielectric function of the films deposited on $\mathrm{Si}$ substrate was spectrally investigated by performing Variable Angle Spectroscopic Ellipsometry (VASE) over the range $350 \mathrm{~nm}<\lambda$ $<1700 \mathrm{~nm}$.

In principle, the two key parameters for TCFs, which are light transmission across the spectral range of interest, and electrical conductivity, grow at the expense of each other. Therefore, a tradeoff between them exists, and a proper Figure of Merit (FOM) for TCFs can be introduced to provide quantitative comparison of performances for TCFs. Among the various evaluation methods [24, 25], the most popular is the FOM proposed by Haacke [26],

$$
\Psi(\lambda)=T(\lambda)^{10} / R_{S}
$$

where $T(\lambda)$ is the transmittance and $R_{S}$ the sheet resistance of the film. Higher values of $\Psi(\lambda)$ represent better transparent-conductive performances.

\section{Results and discussion}

The cross-sectional HR-SEM images of samples S1 and S2 deposited on Si substrate are shown in Figure 1, which highlights the compactness, smoothness of the films, and good adhesion of films to the substrate. Film thickness $(d)$, evaluated from SEM cross-sections was confirmed by step profilometry. Results are listed in Table 1 , with an uncertainty of about $5 \mathrm{~nm}$ caused by the inherent set of electron-beam radius and angular precision of sample orienting during the measurements.
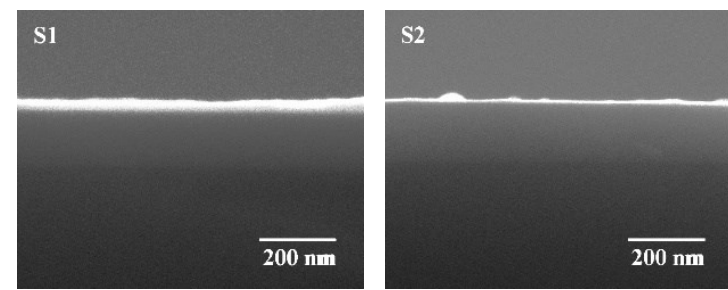

Fig. 1. SEM cross-sections of S1 and S2 films.

Figure 2 illustrates the AFM topographic 3D maps of samples S1-S4, which somehow look equivalent. Very low values for arithmetic mean roughness $\left(R_{a}\right)$ were obtained, indicating extremely smooth surface conditions for these samples. Detailed results are referred in Table 1.
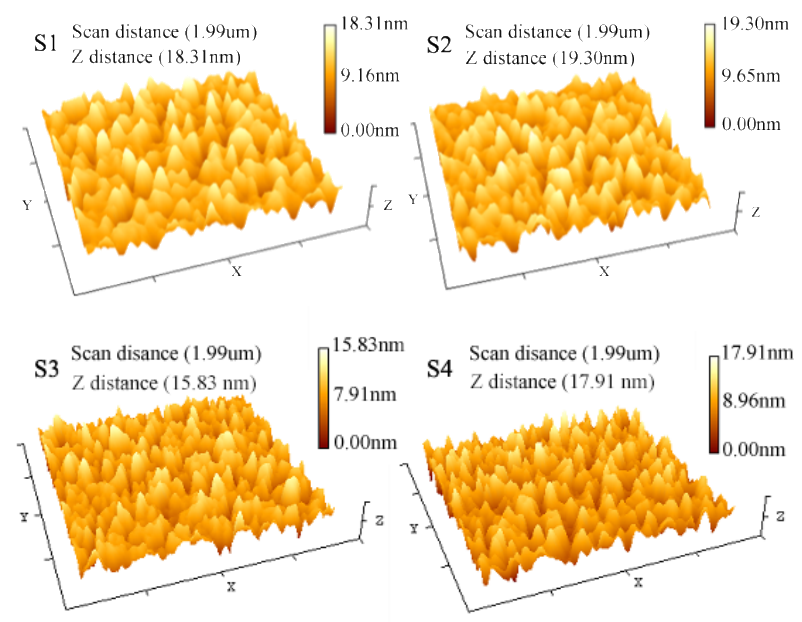

Fig. 2. AFM topographic 3D maps of samples.

The amorphous nature of samples is shown by the smooth XRD patterns in Figure 3. The broad peaks at about $2 \theta=24^{\circ}$ indicate a lack of long-range ordering in $\mathrm{WO}_{3-x}$ lattices. The broad hump at about $70^{\circ}$ is instead originated by scattering of Si (100) substrate.

By XPS investigation of the $\mathrm{W}$ core-line, spectral peaks of $\mathrm{W}^{6+}$ deriving from $\mathrm{WO}_{3}$ phase are detected (spin orbit components $4 \mathrm{f}_{7 / 2}$ and $4 \mathrm{f}_{5 / 2}$ ), as well as peaks that can be assigned to the $4 \mathrm{f}_{7 / 2}$ and $4 \mathrm{f}_{5 / 2}$ of $\mathrm{W}^{+(6-\mathrm{x})}$ ions that derive from sub-stoichiometric $\mathrm{WO}_{3-x}$ phases and oxygen vacancies. Oxygen deficiency and related defects bring intrinsic $n$-type doping and increase electrical conductance of $\mathrm{WO}_{3-x}$ phases, both crystalline $[14,15]$ 
and amorphous [17,27]. Therefore, we expected films to be somewhat conductive. Hot probe measurement on asdeposited samples proved the $n$-type semiconducting nature of $\mathrm{WO}_{3-x}$ samples. Values of sheet resistance $R_{s}$ obtained by 4-probe method are listed in Table 1.

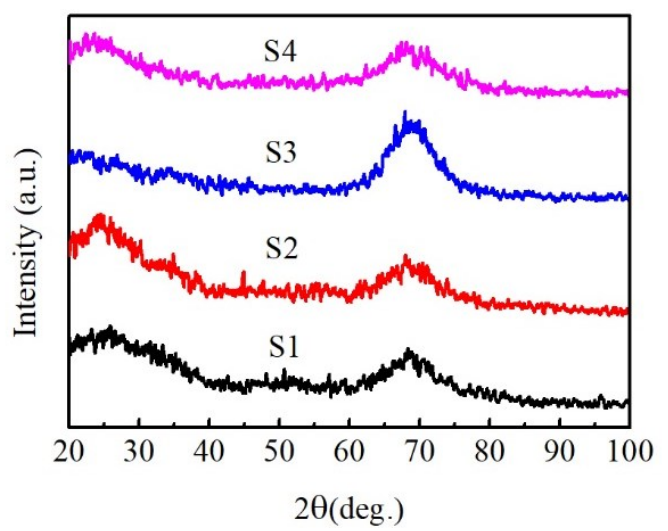

Fig. 3. XRD plots showing the amorphous structure of films.

By considering electrical conductivity at RT to be mainly contributed by band electrons, the $n$-type character of $\mathrm{WO}_{3-x}$ can be correlated to the position of the Fermi level $E_{F}$ and to the value of work function $(\Phi)$ as measured by KP. As $\Phi$ increases, the distance in energy of the Fermi level from the bottom of conduction band $\left(E_{c}-E_{F}\right)$ also increases and the density of free electrons $N$ exponentially decreases, causing resistivity $\rho$ to increase, as $\rho=(N \mu e)^{-1}$, where $\mu$ is the charge mobility, and $e$ is the elementary charge. This is evident for the thicker set of samples, that by annealing both $\Phi$ and $\rho$ increase. However, the KP analysis is not in consistency with the results of electrical conductivity for thicker samples using the previous theory. Instead, samples by annealing show a mild enhancement of electrical conductivity when a higher $\Phi$ is measured comparing to the as-deposited ones.

Table 1. Morphological, electrical, and optical properties of samples S1-S4.

\begin{tabular}{|c|c|c|c|c|}
\hline Sample & S1 & S2 & S3 & S4 \\
\hline $\begin{array}{c}\text { Annealing } \\
\left({ }^{\circ} \mathrm{C}\right)\end{array}$ & No & 300 & No & 300 \\
\hline$d(\mathrm{~nm})$ & $162 \pm 5$ & $159 \pm 5$ & $43 \pm 5$ & $58 \pm 5$ \\
\hline$R_{a}(\mathrm{~nm})$ & 1.83 & 1.82 & 1.59 & 1.80 \\
\hline$\rho(\Omega \cdot \mathrm{cm})$ & $9.47 \mathrm{E}-2$ & $5.10 \mathrm{E}-2$ & 1.26 & 525.48 \\
\hline$R_{s}\left(\Omega \cdot \mathrm{sq}^{-1}\right)$ & $5.85 \mathrm{E}+3$ & $3.21 \mathrm{E}+3$ & $2.93 \mathrm{E}+5$ & $9.06 \mathrm{E}+7$ \\
\hline$\Phi(e V)$ & 4.27 & 4.65 & 4.22 & 4.57 \\
\hline$E_{g}(\mathrm{eV})$ & 2.57 & 2.84 & 2.49 & 3.05 \\
\hline$\alpha @ 1550 \mathrm{~nm}$ & 0.89 & 0.76 & 1.18 & 0.34 \\
\hline$\left.n @ 10^{5} \mathrm{~cm}^{-1}\right)$ & -- & -- & 2.99 & 1.99 \\
\hline$\kappa(150 \mathrm{~nm}$ & -- & -- & 0.46 & $3.42 \mathrm{E}-9$ \\
\hline
\end{tabular}

The slight decrease in sheet resistance values with annealing can be explained in terms of a reduction in the density of grain boundaries and extended defects, which increases the scattering time for charges and charge mobility, thus compensating for the decrease of $N$.

Optical absorption coefficient $\alpha\left(\mathrm{cm}^{-1}\right)$, as retrieved from the absorbance $(A)$ spectra as $\alpha=2.303 A / d$ is reported in Figure 4. Post-growth annealing induces a decrease in absorption. The phenomenon is especially evident for the thinner film S4, for which the absorption coefficient is greatly reduced extensively along the whole examined spectral range. In particular, the absorption coefficient for S4 steadily decreases in the NIR and eventually reaches a value that is smaller than those of the other samples. Optical bandgap $E_{g}(\mathrm{eV})$ values were retrieved by implementing Tauc plot analysis based on the theory of Davis and Mott [28],

$$
(\alpha h v)^{1 / \beta}=B\left(h v-E_{g}\right)
$$

where $h v$ is the photon energy, $B$ is a constant, and $\beta$ is the index accounting for the nature of optical band-toband transitions. The Tauc plot, as shown in the inset of Figure 4, agrees with the results reported elsewhere [5], both in terms of optical bandgap values for substoichiometric $\mathrm{WO}_{3-x}$ and of the indirect nature of interband transitions.

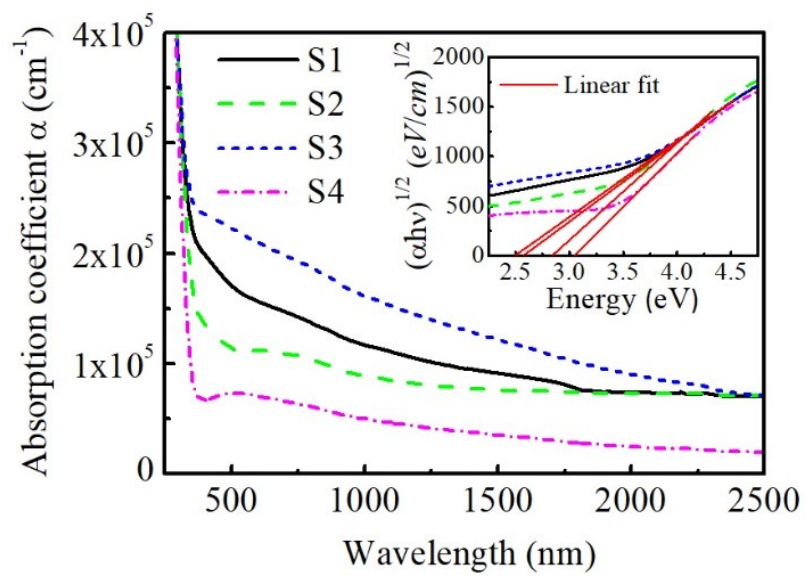

Fig. 4. Absorption coefficient in the visible to NIR range, with the Tauc plot analysis in the inset.

Figure 5 shows the dispersion of real and imaginary parts of the refractive index $(n(\lambda), \kappa(\lambda))$ by VASE. It is interesting that the as-deposited sample $\mathrm{S} 3$ shows a metallike anomalous dispersion within the examined spectral range. Instead, annealed sample S4 shows the normal chromatic dispersion, which is typical of dielectric materials.

Figure 6 compares Haacke's FOM calculated as a function of optical wavelength, for several TCFs and for $\mathrm{WO}_{3-x}$ films in present work. Generally, the good performance of TCFs is limited to around $1.5 \mu \mathrm{m}$, above which the satisfactory FOM becomes invalid. In present work, however, tungsten oxide shows continuous and stable enhancement of FOM along wavelength up to $2.5 \mu \mathrm{m}$. Though the performance within visible range is poor, tungsten oxide outrides the other TCFs when wavelength comes to above $2 \mu \mathrm{m}$. This implies that 
tungsten oxide can be modulated as a promising transparent-conductive oxide specialized for NIR range.
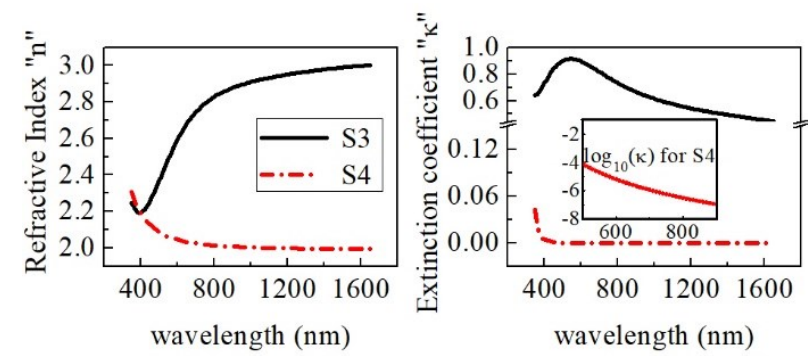

Fig. 5. Spectral dispersion of refractive index (n) and extinction coefficient $(\kappa)$ from VASE. In the right an inset is added to show the $\log _{10}(\kappa)$.

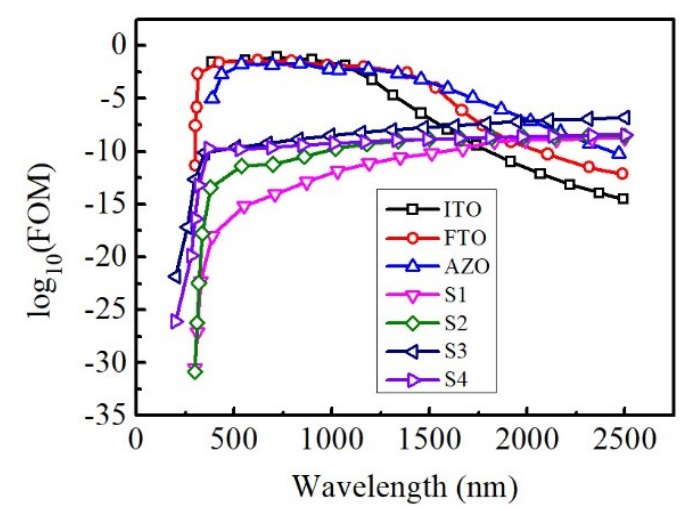

Fig. 6. Figure of merit for different transparent-conductive films, plotted by retrieving transmittance data and sheet resistance from: ITO [29], AZO [29], FTO [30].

\section{Conclusions}

Smooth and compact amorphous tungsten oxide thin films are fabricated by non-reactive RF-sputtering from a $\mathrm{WO}_{3}$ target, as optical layers of tailorable dielectric properties. As-deposited $\mathrm{WO}_{3-x}$ films show good optical absorption and electrical conduction. Post-growth treatment at $300^{\circ} \mathrm{C}$ anneals out oxygen vacancies, leading to better transparency in the NIR. Effects of annealing on electrical resistivity, however, differs for thicker (about $160 \mathrm{~nm}$ ) and thinner films (about $60 \mathrm{~nm}$ ). Electrical resistivity increases by annealing for thicker samples, while for thinner counterparts, electrical resistivity decreases by annealing. Figure of merit, that evaluates the transparent-conductive performance of $\mathrm{WO}_{3-x}$ films, has been analyzed and compared with some popular and commercial transparent-conductive films. Result shows the shortage of $\mathrm{WO}_{3-x}$ as transparent-conductive films in visible range, but very good performance in NIR range.

\section{Acknowledgement}

Authors acknowledge co-funding of research by European fund - FESR, PON Ricerca e Innovazione 20142020.

\section{References}

1. B. Y. Oh et al., J. Appl. Phys. 99, 124505 (2006).

2. H. Kim et al., J. Appl. Phys. 86, 6451 (1999).

3. F. U. Hamelmann, J. Phys. Conf. Ser. 559, 012016 (2014).

4. C. M.Lampert, Sol. Energy Mater. 11, 1 (1984).

5. D. S. Ginley, C. Bright, and G. Editors, MRS Bull. 25, 15 (2000).

6. B. G. Lewis and D. C. Paine, MRS Bull. 25, 22 (2000).

$7 . \quad$ L. Yu, C. Shearer, and J. Shapter, Chem. Rev. 116, 13413 (2016).

8. T. Minami, Thin Solid Films 516, 5822 (2008).

9. A. E. Rakhshani, Y. Makdisi, and H. A. Ramazaniyan, J. Appl. Phys. 83, 1049 (1998).

10. W. Cao et al., J. Photonics Energy 4, 040990 (2014).

11. K. Ellmer, J. Phys. D. Appl. Phys. 33, (2000).

12. A. Al Mohammad and M. Gillet, Thin Solid Films 408, 302 (2002).

13. E. Salje and K. Viswanathan, Acta Crystallogr. Sect. A 31, 356 (1975).

14. D. B. Migas et al., J. Appl. Phys. 108, 093713 (2010).

15. D. B. Migas, V. L. Shaposhnikov, and V. E. Borisenko, J. Appl. Phys. 108, 093714 (2010).

16. F. Wang, C. Di Valentin, and G. Pacchioni, Phys. Rev. B - Condens. Matter Mater. Phys. 84, 1 (2011).

17. F. Mehmood et al., J. Appl. Phys. 120, (2016).

18. A. Vinante et al., Phys. Rev. Lett. 125, 100404 (2020).

19. A. Chiasera et al., Opt. Mater. (Amst). 87, 107 (2019).

20. C. Y. Kim and S. Park, Asian J. Chem. 25, 5874 (2013).

21. A. Axelevitch and G. Golan, Facta Univ. - Ser. Electron. Energ. 26, 187 (2013).

22. L. J. van der Pauw, Philips Res. Reports 13, 1 (1958).

23. J. Tauc, A. Menth, and D. L. Wood, Phys. Rev. Lett. 25, 749 (1970).

24. K. Ellmer, Nat. Photonics 6, 809 (2012).

25. V. K. Jain and A. P. Kulshreshtha, Sol. Energy Mater. 4, 151 (1981).

26. G. Haacke, J. Appl. Phys. 47, 4086 (1976).

27. H. Simchi et al., J. Alloys Compd. 617, 609 (2014).

28. E. A. Davis and N. F. Mott, Philos. Mag. A J. Theor. Exp. Appl. Phys. 22, 903 (1970).

29. D. Langley et al., Nanotechnology 24, (2013).

30. N. Noor and I. P. Parkin, J. Mater. Chem. C 1, 984 (2013). 